Document downloaded from:

http://hdl.handle.net/10251/64755

This paper must be cited as:

Bastin, F.; Conejero Casares, JA.; Esser, C.; Seoane-Sepulveda, JB. (2015). Algebrability and nowhere Gevrey differentiability. Israel Journal of Mathematics. 205:127-143. doi:10.1007/s11856-014-1104-1.

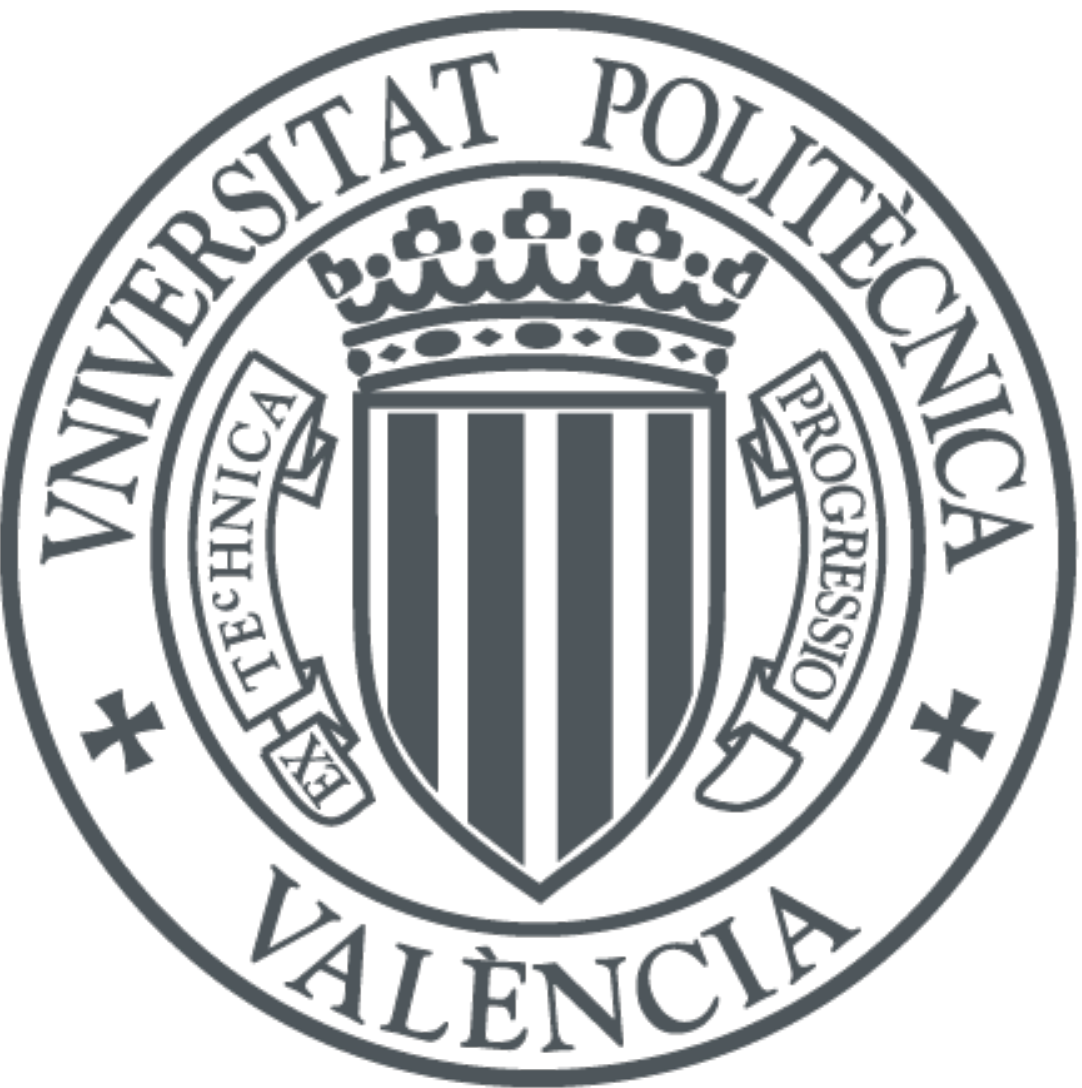

The final publication is available at

http://link.springer.com/article/10.1007/s11856-014-1104-1

Copyright Springer Verlag (Germany)

Additional Information

"The final publication is available at Springer via http://dx.doi.org/10.1007/s11856-014-1104$1 "$ 


\title{
ALGEBRABILITY AND NOWHERE GEVREY DIFFERENTIABILITY
}

\author{
F. BASTIN, J.A. CONEJERO, C. ESSER, AND J.B. SEOANE-SEPÚLVEDA
}

\begin{abstract}
We show that there exist $\mathfrak{c}$-generated algebras (and dense in $\mathcal{C}^{\infty}([0,1])$ ) every nonzero element of which is a nowhere Gevrey differentiable function. This leads to results of dense algebrability (and, therefore, lineability) of functions enjoying this property. In the process of proving these results we also provide a new construction of nowhere Gevrey differentiable functions.
\end{abstract}

\section{INTRODUCTION AND PRELIMINARIES}

The work presented here is a contribution to the ongoing search for large algebraic structures of functions on $[0,1]$ or $\mathbb{R}$ enjoying special properties. Given such a property, we say that the subset $M$ of functions which satisfies it is lineable if $M \cup\{0\}$ contains an infinite dimensional linear (not necessarily closed) space. The concept of lineability was coined by V. I. Gurariy and it first appeared in [1]. In a more general framework we have the following.

Definition 1.1 (Lineability, [1]). Let $X$ be a topological vector space, $M$ a subset of $X$, and $\kappa$ a cardinal number.

(1) $M$ is said to be $\kappa$-lineable if $M \cup\{0\}$ contains a vector space of dimension $\kappa$. At times, we shall be referring to the set $M$ as simply lineable if the existing subspace is infinite dimensional.

(2) We also let $\lambda(M)$ be the maximum cardinality (if it exists) of such a vector space.

(3) When the above linear space can be chosen to be dense in $X$ we shall say that $M$ is $\kappa$-dense-lineable (or, simply, dense-lineable if $\kappa$ is infinite).

Let us recall that (keeping the same notation as in the previous definition) we shall also say that $M$ is spaceable ([1]) if $M \cup\{0\}$ contains an infinite dimensional closed subspace of $X$.

Remark 1.2. (a.) Let us recall that the $\lambda(M)$ from Definition 1.1 might actually not exist. It is not difficult to provide natural examples of sets which are $n$-lineable for every $n \in \mathbb{N}$ but which are not lineable. For instance, let $j_{1} \leq k_{1}<j_{2} \leq \cdots \leq$ $k_{m}<j_{m+1} \leq \ldots$ be positive integers and let $M=\cup_{m}\left\{\sum_{i=j_{m}}^{k_{m}} a_{i} x^{i}: a_{i} \in \mathbb{R}\right\}$. Since

2010 Mathematics Subject Classification. 15A03, 46E10, 26E05, 37C20.

Key words and phrases. Lineability, algebrability, nowhere Gevrey differentiable function.

The second author was supported by MTM2010-14909. The third author was supported by a grant of Research Fellow from the Fonds National de la Recherche Scientifique (FNRS). The second author also wish to thank Programa de Investigación y Desarrollo de la UPV, referencia SP20120700. 
the sets $\left\{\sum_{i=j_{m}}^{k_{m}} a_{i} x^{i}: a_{i} \in \mathbb{R}\right\}(m \in \mathbb{N})$ are pairwise disjoint, $M$ is finitely (but not infinitely) lineable in $\mathcal{C}([0,1])$, the set of continuous functions in $[0,1]$.

(b.) Let us recall that, in [8], the authors introduced the lineability number of a set $M$ as follows

$$
L(M)=\min \{\kappa: M \text { is not } \kappa-\text { lineable }\} .
$$

This number always exists and $L(M)=\lambda(M)^{+}$(the successor cardinal of $\lambda(M)$ ).

Since this concept appeared, it has attracted the attention of many authors, who became interested in the study of subsets of $\mathbb{R}^{\mathbb{R}}$ enjoying certain special or, as they sometimes are called, "pathological" properties (see, e.g., $[1,11,13-15,19]$ and references therein). Before the publication of [1], several authors (when working with infinite dimensional spaces) already found large linear structures enjoying these type of special properties (even though they did not explicitly use the word lineability). We believe that the earliest result in this direction (although negative!) was due to Levine and Milman (1940, [27]):

Theorem 1.3. The subset of $\mathcal{C}([0,1])$ of all functions of bounded variation is not spaceable.

On the other hand, in 1966, Gurariy [23] obtained the following (positive) result within the framework of continuous nowhere differentiable functions (Weierstrass' monsters).

Theorem 1.4. The set of continuous nowhere differentiable functions on $[0,1]$ is lineable.

Afterwards, Fonf, Gurariy, and Kadeč [20] showed that the infinite dimensional subspace from Theorem 1.4 can be chosen to be closed in $\mathcal{C}([0,1])$. As a matter of fact, RodríguezPiazza [29] showed that the space constructed in [20] can also be chosen to be isometrically isomorphic to any separable Banach space. More recently, Hencl [25] showed that any separable Banach space is isometrically isomorphic to a subspace of $\mathcal{C}([0,1])$ whose nonzero elements are nowhere approximately differentiable and nowhere Hölder. We refer the interested reader to the recent expository paper [13] where many more examples can be found and the state of the art of this trend is presented.

Let us also recall that, recently, Bernal [12] introduced the notion of maximal lineable (and that of maximal dense-lineable) meaning that, when keeping the above notation, the dimension of the existing linear space is equal to $\operatorname{dim}(X)$. Besides asking for linear spaces one could also study other structures, such as algebras, which motivated the following concept.

Definition 1.5 (Algebrability and Strong-algebrability, [3,4] and [7]). Given an algebra $\mathcal{A}$ and a subset $\mathcal{B} \subset \mathcal{A}$, we say that:

(1) $\mathcal{B}$ is algebrable if there is a subalgebra $\mathcal{C}$ of $\mathcal{A}$ so that $\mathcal{C} \subset \mathcal{B} \cup\{0\}$ and the cardinality of any system of generators of $\mathcal{C}$ is infinite.

(2) When having $\mathcal{A}$ endowed with a topology, we would say that $\mathcal{B}$ is dense-algebrable if (in addition) $\mathcal{C}$ can be taken dense in $\mathcal{A}$.

(3) At times we shall say that $\mathcal{B}$ is, simply, $\kappa$-algebrable if there exists a $\kappa$-generated subalgebra $\mathcal{C}$ of $\mathcal{A}$ with $\mathcal{C} \subset \mathcal{B} \cup\{0\}$ (where $\kappa$ is some cardinal number).

(4) We also say that $\mathcal{B}$ is strongly $\kappa$-algebrable if there exists a $\kappa$-generated free algebra $\mathcal{C}$ contained in $\mathcal{B} \cup\{0\}$. 
Of course, any algebrable set is, automatically, lineable as well. In general, the converse is false. An example of this can be the set of (improper) Riemann integrable functions on $\mathbb{R}$ (see, e.g., [30]) that are not Lebesgue integrable, denoted $\mathcal{R}(\mathbb{R}) \backslash \mathcal{L}(\mathbb{R})$. This set is lineable (see [22]) but it is also clearly not algebrable. Indeed, for every $f \in \mathcal{R}(\mathbb{R})$, either $f^{2} \notin \mathcal{R}(\mathbb{R})$ or $f^{2}=\left|f^{2}\right| \in \mathcal{R}(\mathbb{R})$ and, therefore, $f^{2} \in \mathcal{L}(\mathbb{R})$. Some of the first examples of algebrable sets appeared in $[4,10]$.

Remark 1.6. As we did in Remark 1.2, (b.), one could also define the following algebrability number:

$$
\min \{\kappa: M \text { is not } \kappa-\text { algebrable }\} .
$$

Of course, the same definition can also be used for strong-algebrability.

Here we shall focus on a very particular class of functions, the so called nowhere Gevrey differentiable functions. In what follows, $\mathcal{C}^{\infty}([0,1])$ denotes the Fréchet space of the functions of class $\mathcal{C}^{\infty}$ on $[0,1]$, endowed with the sequence $\left(p_{k}\right)_{k \in \mathbb{N}_{0}}$ of semi-norms defined by

$$
p_{k}(f)=\sup _{j \leq k} \sup _{x \in[0,1]}\left|f^{(j)}(x)\right|
$$

or, equivalently, with the distance $d$ defined by

$$
d(f, g)=\sum_{k=0}^{+\infty} 2^{-k} \frac{p_{k}(f-g)}{1+p_{k}(f-g)} .
$$

Following [17] we have:

Definition 1.7 (Gevrey differentiable function). For a real number $s>0$ and an open subset $\Omega$ of $\mathbb{R}$ an infinitely differentiable function $f$ in $\Omega$ is said to be Gevrey differentiable of order $s$ at $x_{0} \in \Omega$ if there exist a compact neighborhood $I$ of $x_{0}$ and constants $C, h>0$ such that

$$
\sup _{x \in I}\left|f^{(n)}(x)\right| \leq C h^{n}(n !)^{s}, \quad \forall n \in \mathbb{N} \cup\{0\} .
$$

Clearly, if a function is Gevrey differentiable of order $s$ at $x_{0}$, it is also Gevrey differentiable of any order $s^{\prime}>s$ at $x_{0}$ (the case $s=1$ corresponds to analyticity). On the other hand:

Definition 1.8 (nowhere Gevrey differentiable function). A function $f$ is said to be nowhere Gevrey differentiable (NG from now on) on $\mathbb{R}$ if $f$ is not Gevrey differentiable of order $s$ at $x_{0}$, for every $s>1$ and every $x_{0} \in \mathbb{R}$.

Recall that (following [26]) a Borel set $B$ in a complete metric linear space $E$ is said to be shy if there exists a Borel probability measure $\mu$ on $E$ with compact support such that $\mu(B+x)=0$ for any $x \in E$. A set is said to be prevalent if it is the complement of a shy set. Also, if $X$ is a Baire space, then a subset $A \subset X$ is called residual (or comeager) if $A$ contains some dense $G_{\delta}$ subset of $X$.

Any nowhere Gevrey differentiable function is, in particular, nowhere analytic. The set of nowhere analytic functions in $\mathcal{C}^{\infty}([0,1])$ is known to be prevalent $([9])$, residual $([28])$, lineable $([11])$, and even algebrable $([18])$. In [9] it was also shown that the set of nowhere Gevrey differentiable functions in $\mathcal{C}^{\infty}([0,1])$ is 
(i) a prevalent subset of $\mathcal{C}^{\infty}([0,1])$ and

(ii) a residual subset of $\mathcal{C}^{\infty}([0,1])$.

Thus (in [9]) the authors obtained "genericity" in both the measure-theoretical and the topological senses. On the other hand nothing is known about the algebraic structure of the set NG. One might think that since NG enjoys such a rich Borel structure, it might also contains large algebraic structures (linear spaces, algebras, etc.) This is, in general, not true. For instance, in $[24]$ it was proved that if $\widehat{\mathcal{C}}([0,1])$ denotes the subset of $\mathcal{C}([0,1])$ composed by the functions that attain the maximum exactly once in $[0,1]$, then $\lambda(\widehat{\mathcal{C}}([0,1]))=1$ and, contrary to what one might expect, $\widehat{\mathcal{C}}([0,1])$ is a dense $G_{\delta}$ subset of $\mathcal{C}([0,1])$ (see $[16$, Proposition A]). Thus, there is no immediate implication between being residual and containing large subspaces.

In this paper we shall settle this question for the set of nowhere Gevrey differentiable functions. First of all, we give a direct proof of the maximal-dense-lineability of NG in $\mathcal{C}^{\infty}([0,1])$ (Section 2). To achieve this result we use any nowhere Gevrey differentiable function (see for example [9] for an explicit construction). However, to tackle the problem of algebrability, a more precise knowledge of a very particular "key" function in NG is needed. Following some ideas from $[17,18]$ we are able construct a (real valued) infinitely differentiable nowhere Gevrey differentiable function. This construction allows us to prove the maximal-dense-algebrability of the set of nowhere Gevrey differentiable functions in $\mathcal{C}^{\infty}([0,1])$ (Section 3). We also obtain that $\lambda(\mathrm{NG})=\mathfrak{c}$ (the continuum), which is the best possible result in terms of dimension since the set of continuous functions has cardinality c.

Throughout this paper $C_{j}^{k}$ denotes the binomial coefficient $\frac{j !}{k !(j-k) !}, \mathbb{N}$ is the set of strictly positive natural numbers and $\mathbb{N}_{0}=\mathbb{N} \cup\{0\}$. Also, $\lfloor x\rfloor$ stands for the largest integer smaller than $x$. The rest of the notation shall be rather usual.

\section{Algebrability of NG}

The aim of this section is to prove that the set NG is, both, strongly-algebrable and dense-lineable in $\mathcal{C}^{\infty}([0,1])$ and that $\lambda(\mathrm{NG})=\mathfrak{c}$. This result is, of course, a consequence of the dense-algebrability of NG in $\mathcal{C}^{\infty}([0,1])$ (section 3$)$. Nevertheless, the dense-lineability is here directly obtained, using any function belonging to NG; this is the reason why we show it here as well, to illustrate the differences that one might encounter when dealing with dense-lineability and dense-algebrability.

Proposition 2.1. For every $\alpha \in \mathbb{R}$, let $e_{\alpha}(x)=\exp (\alpha x), x \in \mathbb{R}$. If $f$ is nowhere Gevrey differentiable on $\mathbb{R}$, if $a_{1}, \ldots, a_{N} \in \mathbb{C}$ are not all equal to 0 and if $\alpha_{1}<\cdots<\alpha_{N}$ are real numbers, then the function

$$
g=\sum_{j=1}^{N} a_{j} f e_{\alpha_{j}}
$$

is nowhere Gevrey differentiable on $\mathbb{R}$. It follows that NG is strongly-algebrable. 
The proof of this previous result employs the so-called exponential-like function method. This method was used in [21], rediscovered in [5] and, recently, studied in depth in [6]. Using the fact that the composition of Gevrey functions is still Gevrey (see [31]) and following the lines of the proof of [6, Theorem 5.10], as well as the functions given in the statement of the Theorem, the result follows (we spare the details of its proof to the interested reader, since in Section 3 we shall give an improvement of this result).

Of course, as an immediate corollary, we have:

Corollary 2.2. NG is lineable in $\mathcal{C}^{\infty}([0,1])$.

Lemma 2.3. If $\mathcal{P}$ denotes the set of polynomials, then $\mathcal{P}+\mathrm{NG} \subset \mathrm{NG}$.

Proof. Let us consider $g \in \mathrm{NG}$ and $P$ a polynomial. We proceed by contradiction. Assume that $g+P$ is Gevrey differentiable of order $s>0$ at $x_{0} \in \mathbb{R}$. Since $P$ is analytic at $x_{0}, \mathrm{P}$ is also Gevrey differentiable of order $s$ at $x_{0}$ and the same holds for $g=(g+P)-P$ hence a contradiction.

In order to obtain the dense-lineability of $\mathrm{NG}$ in $\mathcal{C}^{\infty}([0,1])$ let us recall the following result.

Proposition 2.4 (Theorem 2.2 and Remark 2.5, [2]). Let $X$ be a metrizable topological vector space and consider two subsets $A, B$ of $X$ such that $A$ is lineable and $B$ is denselineable in $X$. If $A+B \subset A$, then $A$ is dense-lineable in $X$.

With this result at hand, we can now infer the following.

Proposition 2.5. The set $\mathrm{NG}$ is dense-lineable in $\mathcal{C}^{\infty}([0,1])$.

Proof. It follows directly from Corollary 2.2, Lemma 2.3, and Proposition 2.4.

Next, let us show that the lineability dimension of NG is the largest possible one.

Proposition 2.6. $\lambda(\mathrm{NG})=\mathfrak{c}$.

Proof. Let us fix a function $f \in$ NG. As before, we consider

$$
\mathcal{D}=\operatorname{span}\left\{f e_{\alpha}: \alpha \in[0,1]\right\},
$$

where $e_{\alpha}(x)=\exp (\alpha x)$. From Proposition 2.1, we just have to show that $\operatorname{dim} \mathcal{D}=\mathfrak{c}$. For this, it suffices to show that the functions $f e_{\alpha}, \alpha \in[0,1]$, are linearly independent. Let us assume that it is not the case. Then there exist $c_{1}, \cdots, c_{N} \in \mathbb{C}$ not all zero, and $\alpha_{1}<\cdots<$ $\alpha_{N}$ in $[0,1]$ such that $c_{1} f e_{\alpha_{1}}+\cdots+c_{N} f e_{\alpha_{N}}=0$ on [0,1], i.e., $f\left(c_{1} e_{\alpha_{1}}+\cdots+c_{N} e_{\alpha_{N}}\right)=0$ on $[0,1]$. Since the functions $e_{\alpha_{1}}, \cdots, e_{\alpha_{N}}$ are linearly independent ([11, Theorem 3.1]), there exists $x \in[0,1]$ such that $c_{1} e_{\alpha_{1}}(x)+\cdots+c_{N} e_{\alpha_{N}}(x) \neq 0$. By continuity, there exists a subinterval $J \subset[0,1]$ such that $c_{1} e_{\alpha_{1}}+\cdots+c_{N} e_{\alpha_{N}} \neq 0$ on $[0,1]$. It follows that $f=0$ on $J$, which is impossible since $f$ is nowhere Gevrey differentiable. 


\section{Dense-Algebrability of NG}

The strategy to tackle the algebrability problem will be different from that of the previous section. Here, we shall need a very particular NG function. We can achieve this (see Proposition 3.1) by means of a function defined as a series, in which the $n^{\text {th }}$ term is built via a special function which is Gevrey differentiable of order $n$ on $\mathbb{R}$.

For any $s>1$, let $f_{s}$ denotes the function defined on $\mathbb{R}$ by

$$
f_{s}(x)= \begin{cases}\exp \left(-x^{-\frac{1}{s-1}}\right) & \text { if } x>0 \\ 0 & \text { otherwise. }\end{cases}
$$

In [17], it is proved that $f_{s}$ is Gevrey differentiable of order $s$ on $\mathbb{R}$. Let us consider the function $\psi_{s}$ defined on $\mathbb{R}$ by

$$
\psi_{s}(x)=f_{s}(x) f_{s}(1-x) .
$$

The function $\psi_{s}$ is Gevrey differentiable of order $s$ on $\mathbb{R}$, analytic on ]0,1[, the support of $\psi_{s}$ is $[0,1]$ and $D^{p} \psi_{s}(0)=D^{p} \psi_{s}(1)=0$ for every $p \in \mathbb{N}_{0}$ (i.e. $\psi$ is flat at 0 and 1 ). Consequently, for every $n \geq 2$, there exist $D_{n}>0$ and $h_{n}>0$ such that

$$
\sup _{x \in \mathbb{R}}\left|D^{p} \psi_{n}(x)\right| \leq D_{n}\left(h_{n}\right)^{p}(p !)^{n} \quad \forall p \in \mathbb{N}_{0} .
$$

Keeping the previous notation, we have:

Proposition 3.1. The function $\rho$ defined by

$$
\rho(x)=\sum_{n=2}^{+\infty} C_{n} \psi_{n}\left(2^{n} x-\left\lfloor 2^{n} x\right\rfloor\right)
$$

for every $x \in \mathbb{R}$, where $C_{n}=\left(D_{n}\left(h_{n} 2^{n} n !\right)^{n}\right)^{-1}$, is nowhere Gevrey differentiable on $\mathbb{R}$.

Proof. Due to the flatness of $\psi_{n}$ at 0 and 1, the function $x \mapsto \psi_{n}\left(2^{n} x-\left\lfloor 2^{n} x\right\rfloor\right)$ belongs to $\mathcal{C}^{\infty}(\mathbb{R})$ for every $n \geq 2$. Moreover, for every $p$, from the choice of the coefficients $C_{n}$, the series $\sum_{n=2}^{+\infty} C_{n} 2^{n p} \sup _{x \in \mathbb{R}}\left|D^{p} \psi_{n}(x)\right|$ converges. Therefore, we obtain that the function $\rho$ belongs to $\mathcal{C}^{\infty}(\mathbb{R})$.

Let us show that $\rho$ is nowhere Gevrey differentiable. The set $Q$ of all points of the form $2^{-m} k$, where $m \geq 3$ is a natural number and $k$ is an odd number, is dense in $\mathbb{R}$. Therefore, it suffices to show that $\rho$ is not Gevrey differentiable of any order at each point of $Q$. On the contrary, assume that $\rho$ is Gevrey differentiable of order $s>1$ at some point $x_{0} \in Q$. Let $x_{0}=2^{-m_{0}} k_{0}$. Then for $n \in\left\{2, \ldots, m_{0}-1\right\}$, the function $\psi_{n}\left(2^{n} x-\left\lfloor 2^{n} x\right\rfloor\right)$ is analytic at $x_{0}$ and hence Gevrey differentiable of order $s$ at $x_{0}$. Consequently, the function

$$
\Theta_{m_{0}}(x):=\sum_{n=m_{0}}^{+\infty} C_{n} \psi_{n}\left(2^{n} x-\left\lfloor 2^{n} x\right\rfloor\right)=\rho(x)-\sum_{n=2}^{m_{0}-1} C_{n} \psi_{n}\left(2^{n} x-\left\lfloor 2^{n} x\right\rfloor\right)
$$


is also Gevrey differentiable of order $s$ at $x_{0}$. Since $\Theta_{m_{0}}$ is periodic of period $2^{-m_{0}}$, we can assume that $x_{0}=0$. Then, there exist $\varepsilon>0, C>0$ and $h>0$ such that

$$
\sup _{|x| \leq \varepsilon}\left|D^{p} \Theta_{m_{0}}(x)\right| \leq C h^{p}(p !)^{s} \quad \forall p \in \mathbb{N}_{0} .
$$

Since each derivative of $\Theta_{m_{0}}$ at 0 is equal to 0 , Taylor's formula gives that for every $x \in \mathbb{R}$ and every $p \in \mathbb{N}$, there exists a real number $\xi$ between 0 and $x$ such that

$$
\Theta_{m_{0}}(x)=\frac{D^{p} \Theta_{m_{0}}(\xi)}{p !} x^{p}
$$

Then, we have

$$
0 \leq \Theta_{m_{0}}(x) \leq C x^{p} h^{p}(p !)^{s-1} \forall p \in \mathbb{N}, \quad \forall 0<x \leq \varepsilon,
$$

and it follows that

$$
0 \leq C_{n} \psi_{n}\left(2^{n} x-\left\lfloor 2^{n} x\right\rfloor\right) \leq C x^{p} h^{p}(p !)^{s-1}
$$

for every $p \in \mathbb{N}, n \geq m_{0}$ and $0<x \leq \varepsilon$. Let us fix $n$ large enough such that $n \geq s, n \geq m_{0}$ and $h 2^{-n} e<1$. For every $p \in \mathbb{N}$, we define then $x_{p}:=2^{-n} p^{-(n-1)}$. For $p$ sufficiently large, we have $0<x_{p}<\varepsilon$ and we obtain then

$$
0 \leq C_{n} \psi_{n}\left(p^{-(n-1)}\right) \leq C h^{p} 2^{-n p} p^{-p(n-1)}(p !)^{s-1},
$$

where $\psi_{n}\left(p^{-(n-1)}\right)=e^{-p} f_{n}\left(1-p^{-(n-1)}\right)$. Consequently, we have

$$
C_{n} f_{n}\left(1-p^{-(n-1)}\right) \leq C h^{p} 2^{-n p} e^{p}\left(p^{-p} p !\right)^{s-1} .
$$

for every $p$ large enough. The left-hand side converges to $C_{n} f_{n}(1)=C_{n} e^{-1}>0$ and the right-hand side converges to 0 when $p \rightarrow+\infty$. This leads to a contradiction.

The following proposition improves Proposition 2.1. It is the second key of the main result in this section.

Proposition 3.2. If $F_{1}, \ldots, F_{N}$ are analytic on $\mathbb{R}$ and not all identically equal to 0 , and if $\rho$ is the function from Proposition 3.1, then the function

$$
g=\sum_{i=1}^{N} F_{i} \rho^{i}
$$

is nowhere Gevrey differentiable on $\mathbb{R}$.

Proof. As previously, consider the set $Q$ of all points of the form $2^{-m} k$, where $m \geq 3$ is a natural number and $k$ is an odd number. Since $Q$ is dense in $\mathbb{R}$, we just have to show that $g$ is not Gevrey differentiable of any order at each point of $Q$. On the contrary, assume that $g$ is Gevrey differentiable of order $s>1$ at some point $x_{0}=2^{-m_{0}} k_{0}$.

Recall that we do not necessarily have flatness of $\rho$ at $x_{0}$. This is the reason why we set

$$
A_{m_{0}}(x):=\sum_{n=2}^{m_{0}-1} C_{n} \psi_{n}\left(2^{n} x-\left\lfloor 2^{n} x\right\rfloor\right) \text { and } \Theta_{m_{0}}(x):=\sum_{n=m_{0}}^{+\infty} C_{n} \psi_{n}\left(2^{n} x-\left\lfloor 2^{n} x\right\rfloor\right)
$$


for every $x \in \mathbb{R}$. Then, $A_{m_{0}}$ is analytic at $x_{0}$ and that $\Theta_{m_{0}}$ is flat at $x_{0}$. Of course, we also have

$$
\rho=A_{m_{0}}+\Theta_{m_{0}}
$$

and it follows that

$$
\begin{aligned}
g(x) & =\sum_{i=1}^{N} F_{i}(x)\left(A_{m_{0}}(x)+\Theta_{m_{0}}(x)\right)^{i}=\sum_{i=1}^{N} F_{i}(x) \sum_{j=0}^{i} C_{i}^{j}\left(A_{m_{0}}(x)\right)^{i-j}\left(\Theta_{m_{0}}(x)\right)^{j} \\
& =\sum_{i=1}^{N} F_{i}(x)\left(A_{m_{0}}(x)\right)^{i}+\sum_{i=1}^{N} F_{i}(x) \sum_{j=1}^{i} C_{i}^{j}\left(A_{m_{0}}(x)\right)^{i-j}\left(\Theta_{m_{0}}(x)\right)^{j} \\
& =\sum_{i=1}^{N} F_{i}(x)\left(A_{m_{0}}(x)\right)^{i}+\sum_{j=1}^{N}\left(\sum_{i=j}^{N} F_{i}(x) C_{i}^{j}\left(A_{m_{0}}(x)\right)^{i-j}\right)\left(\Theta_{m_{0}}(x)\right)^{j} \\
& =\sum_{i=1}^{N} F_{i}(x)\left(A_{m_{0}}(x)\right)^{i}+\sum_{j=1}^{N} c_{j}(x)\left(\Theta_{m_{0}}(x)\right)^{j},
\end{aligned}
$$

where for every $j \in\{1, \ldots, N\}$

$$
c_{j}(x):=\sum_{i=j}^{N} F_{i}(x) C_{i}^{j}\left(A_{m_{0}}(x)\right)^{i-j} .
$$

Let us fix a neighborhood $V$ of $x_{0}$ and let us show that there exists $j \in\{1, \ldots, N\}$ such that $c_{j}$ is not identically 0 in $V$. We proceed by contradiction. Assume that $c_{j}(x)=0$ for every $j \in\{1, \ldots, N\}$ and $x \in V$. This would mean that

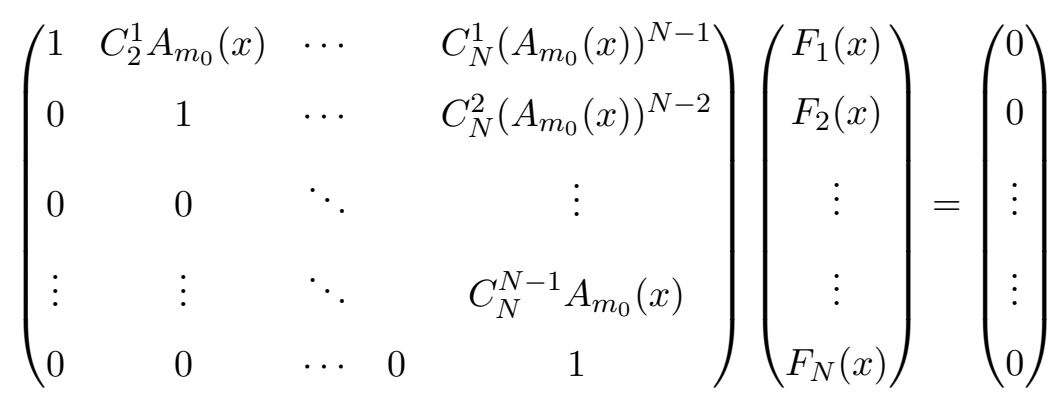

for every $x \in V$. Since $F_{1}, \ldots, F_{N}$ are not all identically equal to 0 , there is $x \in V$ and $j \in\{1, \ldots, N\}$ such that $F_{j}(x) \neq 0$, which gives a contradiction since the matrix is invertible. Let $k$ be the smallest element of $\{1, \ldots, N\}$ for which $c_{k}$ is not identically equal to 0 on $V$. Then, in this neighborhood, we have

$$
g(x)=\sum_{i=1}^{N} F_{i}(x)\left(A_{m_{0}}(x)\right)^{i}+\sum_{j=k}^{N} c_{j}(x)\left(\Theta_{m_{0}}(x)\right)^{j} .
$$


Since $\sum_{i=1}^{N} F_{i}(x)\left(A_{m_{0}}(x)\right)^{i}$ is analytic at $x_{0}$ and since $g$ is Gevrey differentiable of order $s$ at $x_{0}$, we have that the function

$$
\Phi_{m_{0}}(x):=\sum_{j=k}^{N} c_{j}(x)\left(\Theta_{m_{0}}(x)\right)^{j}
$$

is also Gevrey differentiable of order $s$ at $x_{0}$. Then, there exist $\varepsilon>0, C>0$, and $h>0$ such that

$$
\sup _{\left|x-x_{0}\right| \leq \varepsilon}\left|D^{p} \Phi_{m_{0}}(x)\right| \leq C h^{p}(p !)^{s} \quad \forall p \in \mathbb{N}_{0}
$$

From the flatness of $\Theta_{m_{0}}$ at $x_{0}$, we also get that $\Phi_{m_{0}}$ is flat at $x_{0}$. Then, by Taylor's formula, for every $x \in \mathbb{R}$ and every $p \in \mathbb{N}$, there is $\xi$ between $x$ and $x_{0}$ such that

$$
\Phi_{m_{0}}(x)=\frac{D^{p} \Phi_{m_{0}}(\xi)}{p !}\left(x-x_{0}\right)^{p} .
$$

Consequently, we have

$$
\left|\Phi_{m_{0}}(x)\right| \leq C h^{p}(p !)^{s-1}\left|x-x_{0}\right|^{p}
$$

for every $x$ such that $\left|x-x_{0}\right| \leq \varepsilon$ and for every $p \in \mathbb{N}$.

Recall that the function $c_{k}$ is analytic at $x_{0}$ and not identically equal to 0 in a neighborhood of $x_{0}$. Thus, there exists $J \in \mathbb{N}_{0}$ and $d_{k}$ analytic at $x_{0}$ with $d_{k}\left(x_{0}\right) \neq 0$ and such that

$$
c_{k}(x)=\left(x-x_{0}\right)^{J} d_{k}(x)
$$

in a neighborhood of $x_{0}$. Let us fix $n \in \mathbb{N}$ such that $n>s, n \geq m_{0}$ and $h e^{k} 2^{-n}<1$.

As before, we consider $x_{p}:=x_{0}+2^{-n} p^{-\left(s_{0}-1\right)}$ for every $p \in \mathbb{N}$. Then, on one hand, we have

$$
\frac{\Phi_{m_{0}}\left(x_{p}\right)}{\left(\Theta_{m_{0}}\left(x_{p}\right)\right)^{k}\left(x_{p}-x_{0}\right)^{J}}=d_{k}\left(x_{p}\right)+\sum_{j=k+1}^{N} c_{j}\left(x_{p}\right) \frac{\left(\Theta_{m_{0}}\left(x_{p}\right)\right)^{j-k}}{\left(x_{p}-x_{0}\right)^{J}}
$$

which converges to $d_{k}\left(x_{0}\right) \neq 0$ as $p$ goes to infinity (the second term of the sum converges to 0 since $\Theta_{m_{0}}$ is flat at $\left.x_{0}\right)$. On the other hand, for $p$ large enough, we have $\left|x_{p}-x_{0}\right| \leq \varepsilon$ and it follows that

$$
\left|\Phi_{m_{0}}\left(x_{p}\right)\right| \leq C h^{p}(p !)^{s-1}\left|x_{p}-x_{0}\right|^{p}
$$


Moreover, for $p$ large enough, we have $2^{n} x_{p}-\left\lfloor 2^{n} x_{p}\right\rfloor=p^{-(n-1)}$ and $f_{n}\left(1-p^{-(n-1)}\right)$ converges to $f_{n}(1)=e^{-1}>0$ if $p$ goes to infinity. Therefore, we obtain that

$$
\begin{aligned}
\left|\frac{\Phi_{m_{0}}\left(x_{p}\right)}{\left(\Theta_{m_{0}}\left(x_{p}\right)\right)^{k}\left(x_{p}-x_{0}\right)^{J}}\right| & \leq \frac{C h^{p}(p !)^{s-1}\left|x_{p}-x_{0}\right|^{p}}{\left(C_{n} \psi_{n}\left(2^{n} x_{p}-\left\lfloor 2^{n} x_{p}\right\rfloor\right)\right)^{k}\left|x_{p}-x_{0}\right|^{J}} \\
& =\frac{C h^{p}(p !)^{s-1} 2^{-n(p-J)} p^{-(p-J)(n-1)}}{\left(C_{n} e^{-p} f_{n}\left(1-p^{-n-1}\right)\right)^{k}} \\
& \leq \frac{C 2^{n J}}{\left(C_{n} f_{n}\left(1-p^{-n-1}\right)\right)^{k}}\left(\frac{p !}{p^{p}}\right)^{n-1} p^{J(n-1)}\left(h e^{k} 2^{-n}\right)^{p},
\end{aligned}
$$

which converges to 0 as $p$ goes to infinity. This contradiction gives the conclusion.

Let $\mathcal{H}$ denote a Hamel basis of $\mathbb{R}$, let $\mathcal{A}$ be an algebra generated by the functions $\rho e_{\alpha}$ with $\alpha \in \mathcal{H}$ and $e_{\alpha}(x)=\exp (\alpha x)$. Then $f$ of $\mathcal{A}$ if and only if $f$ is of the form

$$
f=\sum_{l=1}^{L} a_{l} \rho^{n_{l}} e_{\beta_{l}}
$$

where $L \in \mathbb{N}, a_{l} \in \mathbb{R}$ for all $l \in\{1, \ldots, L\}$, and $\beta_{l} \neq \beta_{l^{\prime}}$ if $l \neq l^{\prime}$.

Proposition 3.3. $\mathcal{A}$ is a $\mathfrak{c}$-generated free algebra contained in $\mathrm{NG} \cup\{0\}$.

Proof. By Proposition 3.2, $\mathcal{A} \subset \mathrm{NG} \cup\{0\}$. Using the periodicity of $\rho$ and the properties of Vandermonde determinants, we obtain that the functions $\rho^{n_{l}} e_{\beta_{l}}$ are linearly independent.

In order to obtain strongly dense-algebrability of NG, we are now going to modify a little bit the definition of the previous algebra as explained in what follows. First we need some additional notations and a lemma.

Let $\alpha_{m} \in \mathbb{R}(m \in \mathbb{N})$. Using the continuity of the multiplication by scalars, for every $m$, we take $k_{m}>0$ such that $d\left(0, k_{m} e_{\alpha_{m}} \rho\right)<\frac{1}{m}$. Let also $P_{m}(m \in \mathbb{N})$ be a dense sequence of polynomials in $\mathcal{C}^{\infty}([0,1])$.

Lemma 3.4. The family $\mathcal{G}_{0}:=\left\{P_{m}+k_{m} \rho e_{\alpha_{m}}: m \in \mathbb{N}\right\}$ is dense in $\mathcal{C}^{\infty}([0,1])$.

Proof. For every $f \in \mathcal{C}^{\infty}([0,1])$ and for every $m$, we have

$$
d\left(f, P_{m}+k_{m} e_{\alpha_{m}} \rho\right) \leq d\left(f, P_{m}\right)+d\left(0, k_{m} e_{\alpha_{m}} \rho\right) \leq d\left(f, P_{m}\right)+\frac{1}{m} .
$$

Since there is a subsequence $M(k) \in \mathbb{N}(k \in \mathbb{N})$ such that $\lim _{k} d\left(f, P_{M(k)}\right)=0$, we conclude.

Now, take a sequence of different elements $\alpha_{m} \in \mathcal{H}(m \in \mathbb{N})$ and define $k_{\alpha}=1, P_{\alpha}=0$ for $\alpha \in \mathcal{H} \backslash\left\{\alpha_{m}: m \in \mathbb{N}\right\}$. The "candidate" we are looking for is the algebra $\mathcal{A}_{d}$ generated by

$$
\mathcal{G}:=\left\{P_{\alpha}+k_{\alpha} \rho e_{\alpha}: \alpha \in \mathcal{H}\right\}
$$


Theorem 3.5. $\mathcal{A}_{d}$ is a is $\mathfrak{c}$-generated free dense-algebra $\left(\right.$ in $\left.\mathcal{C}^{\infty}([0,1])\right)$ and contained in $\mathrm{NG} \cup\{0\}$.

Proof. On the one hand, since the set of generators $\mathcal{G}$ contains $\mathcal{G}_{0}$, Lemma 3.4 provides the density. On the other hand, the functions $\rho e_{\alpha}\left(\alpha \in \mathcal{H} \backslash\left\{\alpha_{m}: m \in \mathbb{N}\right\}\right)$ are generators; using Proposition 3.3 we obtain the fact that $\mathcal{A}_{d}$ is $\mathfrak{c}$-generated. It remains then to show that $\mathcal{A}_{d} \subset \mathrm{NG} \cup\{0\}$. An element $f \neq 0$ of $\mathcal{A}_{d}$ can be written as

$$
f=\sum_{l=1}^{L} a_{l} \prod_{j=1}^{J}\left(P_{\gamma_{j}}+k_{\gamma_{j}} e_{\gamma_{j}} \rho\right)^{n(l, j)}
$$

where $J, L \in \mathbb{N}, a_{l} \in \mathbb{R} \backslash\{0\}$ for all $l \in\{1, \ldots L\}, \gamma_{j} \in \mathcal{H}$ for all $j \in\{1, \ldots J\}$ (with $\gamma_{j} \neq \gamma_{j^{\prime}}$ if $\left.j \neq j^{\prime}\right)$ and where $n(l, j) \in \mathbb{N}_{0}$ are such that $n(l, j) \neq n\left(l^{\prime}, j\right)$ for at least one $j$ in case $l \neq l^{\prime}$. As before, we set $\beta_{l}:=\sum_{j=1}^{J} n(l, j) \gamma_{j}(l \in\{1, \ldots, L\})$ and we have $\beta_{l} \neq \beta_{l^{\prime}}$ if $l \neq l^{\prime}$.

For each $l \in\{1, \ldots, L\}$, the term

$$
\prod_{j=1}^{J}\left(P_{\gamma_{j}}+k_{\gamma_{j}} e_{\gamma_{j}} \rho\right)^{n(l, j)}
$$

is a "polynomial" (with coefficients which are analytic functions) in the "variable" $\rho$; the "degree" of this polynomial is $n_{l}=\sum_{j=1}^{J} n(l, j) \in \mathbb{N}$ and the coefficient of $\rho^{n_{l}}$ is

$$
c_{l}=\left(\prod_{j=1}^{J} k_{\gamma_{j}}^{n(l, j)}\right) e_{\beta_{l}}
$$

Let $N=\sup \left\{n_{1}, \ldots, n_{L}\right\}$. The function $f$ also appears as a "polynomial" (with coefficients which are analytic functions) in the "variable" $\rho$ and the coefficient of the term with the highest power $N$ is

$$
F_{N}:=\sum_{1 \leq l \leq L, n_{l}=N} a_{l} c_{l}=\sum_{1 \leq l \leq L, n_{l}=N} a_{l}\left(\prod_{j=1}^{J} k_{\gamma_{j}}^{n(l, j)}\right) e_{\beta_{l}} .
$$

Since the coefficients $a_{l}$ are not zero and since the $\beta_{l}$ are different, $F_{N}$ is not identically 0 . Hence the conclusion using Proposition 3.2 and the fact that the sum of a polynomial and a NG function is still a NG function.

We would like to finish by pointing out some remarks. In the existing literature, many examples of continuous functions (enjoying certain pathological properties) were constructing within the framework of $\mathcal{C}([0,1])$.

Of course, when it comes to spaceability, the results may differ very much from one another depending on which subspace of continuous functions we are considering. For instance, in 1966, a classical result by Gurariy [23] states the following. 
Theorem. The set of everywhere differentiable functions on $[0,1]$ is not spaceable in $\mathcal{C}([0,1])$.

On the other hand, Gurariy also proved in [23] that there actually exist closed infinite dimensional subspaces of $\mathcal{C}([0,1])$ all of whose members are differentiable on $] 0,1[$. However, Bernal [11, Theorem 4.4] showed that $\mathcal{C}^{\infty}(] 0,1[)$ is, indeed, spaceable in $\mathcal{C}(] 0,1[)$.

Next, we would like to recall that Proposition 2.5 and Theorem 3.5 in this paper can be easily adapted to the case of nowhere Gevrey differentiable funcions in $\mathcal{C}^{\infty}(\mathbb{R})$ (and not just $[0,1])$, since $\mathcal{C}^{\infty}(\mathbb{R})$ is also a Fréchet space and the polynomials are also dense in it (and, also, employing Theorem 2.2 and Remark 2.5 from [2] as well).

Acknowledgments. The authors would like to thank the referee for his thoroughly analysis of this paper, for Remarks 1.2 (b.) and 1.6, and for pointing out references [5], [6], [7], [8], [21], and [31]. All of this helped in improving the paper and in making it easier to read.

\section{REFERENCES}

[1] R. M. Aron, V. I. Gurariy, and J. B. Seoane-Sepúlveda, Lineability and spaceability of sets of functions on $\mathbb{R}$, Proc. Amer. Math. Soc. 133 (2005), no. 3, 795-803.

[2] R. M. Aron, F. J. García-Pacheco, D. Pérez-García, and J. B. Seoane-Sepúlveda, On dense-lineability of sets of functions on $\mathbb{R}$, Topology 48 (2009), no. 2-4, 149-156.

[3] R. M. Aron, D. Pérez-García, and J. B. Seoane-Sepúlveda, Algebrability of the set of non-convergent Fourier series, Studia Math. 175 (2006), no. 1, 83-90.

[4] R. M. Aron and J. B. Seoane-Sepúlveda, Algebrability of the set of everywhere surjective functions on $\mathbb{C}$, Bull. Belg. Math. Soc. Simon Stevin 14 (2007), no. 1, 25-31.

[5] M. Balcerzak, A. Bartoszewicz, and M. Filipczak, Nonseparable spaceability and strong algebrability of sets of continuous singular functions, J. Math. Anal. Appl. 407 (2013), no. 2, 263-269.

[6] A. Bartoszewicz, M. Bienias, M. Filipczak, and S. Głąb, Exponential-like function method in strong $\mathfrak{c}$-algebrability, arXiv:1307.0331.

[7] A. Bartoszewicz and S. Głąb, Strong algebrability of sets of sequences and functions, Proc. Amer. Math. Soc. 141 (2013), no. 3, 827-835.

[8] A. Bartoszewicz and S. Głąb, Additivity and lineability in vector spaces, Linear Algebra Appl. 439 (2013), no. 7, 2123-2130.

[9] F. Bastin, C. Esser, and S. Nicolay, Prevalence of "nowhere analyticity", Studia Math. 210 (2012), no. 3, 239-246.

[10] F. Bayart and L. Quarta, Algebras in sets of queer functions, Israel J. Math. 158 (2007), 285-296.

[11] L. Bernal-González, Lineability of sets of nowhere analytic functions, J. Math. Anal. Appl. 340 (2008), no. 2, 1284-1295.

[12] _ Algebraic genericity of strict-order integrability, Studia Math. 199 (2010), no. 3, $279-293$.

[13] L. Bernal-González, D. Pellegrino, and J. B. Seoane-Sepúlveda, Linear subsets of nonlinear sets in topological vector spaces, Bull. Amer. Math. Soc. (N.S.) (2014), DOI: http://dx.doi.org/10.1090/S02730979-2013-01421-6.

[14] G. Botelho, D. Cariello, V. V. Fávaro, and D. Pellegrino, Maximal spaceability in sequence spaces, Linear Algebra Appl. 437 (2012), no. 12, 2978-2985.

[15] G. Botelho, D. Cariello, V. V. Fávaro, D. Pellegrino, and J. B. Seoane-Sepúlveda, Distinguished subspaces of $L_{p}$ of maximal dimension, Studia Math. 215 (2013), no. 3, 261-280. 
[16] G. Botelho, D. Cariello, V. V. Fávaro, D. Pellegrino, and J. B. Seoane-Sepúlveda, On very non-linear subsets of continuous functions, Q. J. Math. (2013), in press.

[17] S.-Y. Chung and J. Chung, There exist no gaps between Gevrey differentiable and nowhere Gevrey differentiable, Proc. Amer. Math. Soc. 133 (2005), no. 3, 859-863 (electronic).

[18] J. A. Conejero, P. Jiménez-Rodríguez, G. A. Muñoz-Fernández, and J. B. Seoane-Sepúlveda, When the Identity Theorem "seems" to fail, Amer. Math. Monthly (2013), in press.

[19] P. H. Enflo, V. I. Gurariy, and J. B. Seoane-Sepúlveda, Some Results and Open Questions on Spaceability in Function Spaces, Trans. Amer. Math. Soc. (2013), DOI: http://dx.doi.org/10.1090/S00029947-2013-05747-9.

[20] V. P. Fonf, V. I. Gurariy, and M. I. Kadets, An infinite dimensional subspace of C $[0,1]$ consisting of nowhere differentiable functions, C. R. Acad. Bulgare Sci. 52 (1999), no. 11-12, 13-16.

[21] D. García, B. C. Grecu, M. Maestre, and J. B. Seoane-Sepúlveda, Infinite dimensional Banach spaces of functions with nonlinear properties, Math. Nachr. 283 (2010), no. 5, 712-720.

[22] F. J. García-Pacheco, M. Martín, and J. B. Seoane-Sepúlveda, Lineability, spaceability, and algebrability of certain subsets of function spaces, Taiwanese J. Math. 13 (2009), no. 4, 1257-1269.

[23] V. I. Gurariy, Subspaces and bases in spaces of continuous functions, Dokl. Akad. Nauk SSSR 167 (1966), 971-973 (Russian).

[24] V. I. Gurariy and L. Quarta, On lineability of sets of continuous functions, J. Math. Anal. Appl. 294 (2004), no. 1, 62-72.

[25] S. Hencl, Isometrical embeddings of separable Banach spaces into the set of nowhere approximatively differentiable and nowhere Hölder functions, Proc. Amer. Math. Soc. 128 (2000), no. 12, 3505-3511.

[26] B. R. Hunt, T. Sauer, and J. A. Yorke, Prevalence: a translation-invariant "almost every" on infinitedimensional spaces, Bull. Amer. Math. Soc. (N.S.) 27 (1992), no. 2, 217-238.

[27] B. Levine and D. Milman, On linear sets in space $C$ consisting of functions of bounded variation, Comm. Inst. Sci. Math. Méc. Univ. Kharkoff [Zapiski Inst. Mat. Mech.] (4) 16 (1940), 102-105 (Russian, with English summary).

[28] D. Morgenstern, Unendlich oft differenzierbare nicht-analytische Funktionen, Math. Nachr. 12 (1954), 74 (German).

[29] L. Rodríguez-Piazza, Every separable Banach space is isometric to a space of continuous nowhere differentiable functions, Proc. Amer. Math. Soc. 123 (1995), no. 12, 3649-3654.

[30] R. L. Wheeden and A. Zygmund, Measure and integral, Marcel Dekker Inc., New York, 1977. An introduction to real analysis; Pure and Applied Mathematics, Vol. 43.

[31] T. Yamanaka, A new higher order chain rule and Gevrey class, Ann. Global Anal. Geom. 7 (1989), no. 3, 179-203.

\section{F. BASTIN,}

Institute OF MATHEMATiCs B37

UNIVERSITY OF LIÈGE

B-4000 Liège, Belgium.

E-mail address: F.Bastin@ulg.ac.be

J.A. Conejero,

Instituto Universitario de Matemática Pura y Aplicada

Universitat Politècnica de València

46022, VAlÈnCia, Spain.

E-mail address: aconejero@upv.es 
C. Esser,

Institute of Mathematics B37

UNIVERSITY OF LIÈGE

B-4000 LiÈge, BELGium.

E-mail address: Celine.Esser@ulg.ac.be

J.B. Seoane-Sepúlveda,

Plaza de Ciencias 3

Facultad de Matemáticas

Departamento de AnÁlisis

Universidad Complutense de Madrid, 28040, MAdRID, SPAIN.

E-mail address: jseoane@mat.ucm.es 